\title{
Automated Quantification of Muscle and Fat in the Thigh from Water-, Fat-and Non-Suppressed MR Images
}

\author{
Sokratis Makrogiannis, PhD, Suraj Serai, PhD, Kenneth W. Fishbein, PhD, Catherine \\ Schreiber, MD, Luigi Ferrucci, MD, PhD, and Richard G Spencer, MD, PhD
}

\begin{abstract}
Purpose-To introduce and validate an unsupervised muscle and fat quantification algorithm based on joint analysis of water-suppressed (WS), fat-suppressed (FS) and water-and-fat (nonsuppressed) volumetric MR images of the mid-thigh region.
\end{abstract}

\begin{abstract}
Materials and Methods-We first segment the subcutaneous fat by use of a parametric deformable model, and then apply centroid clustering in the feature domain defined by the voxel intensities in water- and fat-suppressed images to identify the inter-muscular fat and muscle. In the final step we compute volumetric and area measures of fat and muscle. We applied this algorithm on datasets of water-, fat- and non-suppressed volumetric MR images acquired from 28 participants.
\end{abstract}

\begin{abstract}
Results-We validated our tissue composition analysis against fat and muscle area measurements obtained from semi-manual analysis of single-slice mid-thigh CT images of the same participants and found very good agreement between the two methods. Furthermore, we compared the proposed approach with a variant that uses non-suppressed images only and observed that joint analysis of water- and fat-suppressed images is more accurate than the nonsuppressed only variant.
\end{abstract}

Conclusion-Our MRI algorithm produces accurate tissue quantification, is less labor intensive and more reproducible than the original CT-based workflow and can address inter-participant anatomic variability and intensity inhomogeneity effects.

\section{Keywords}

local tissue composition; image segmentation; thigh imaging

\section{INTRODUCTION}

Understanding body composition and the regional distribution of adipose and muscle tissues is a task of central importance for the study of metabolic diseases in humans $(1,2)$. Its significance is further heightened by the growing obesity rates in the U.S. and worldwide (3). Recent reports have shown that changes in body composition-typically observed as an increase in fat mass and reduction of lean body mass- reflect the cumulative effect of aging and chronic diseases and mediate important aspects of their impact on physical function and resiliency (4).

One of the main advantages of MRI in this context is the ability to distinguish between different types of soft tissue (5). Recent MRI techniques that generate water-suppressed (i.e., fat-only) and fat-suppressed (i.e., water-only) images along with spectroscopic methods

Corresponding author: Sokratis Makrogiannis, PhD, 3001 S. Hanover St., 5th fl., National Institute on Aging, National Institutes of Health, Baltimore, Maryland, 21225, Tel: 410-350-8481, Fax: 410-350-3979, makrogianniss@ mail.nih.gov. 
allow computation of muscle area and fat composition (6). Usually the goal is to compute volumetric measures of the muscle and fat, and to separate the adipose tissue into subcutaneous and intermuscular compartments. In the present work, in order to distinguish macroscopic, MRI-resolvable fat deposits embedded between muscle regions from microscopic fat deposits detectable within muscle only by CT or localized MR spectroscopy, we will refer to the former as 'intermuscular fat' and the latter as 'intramuscular fat'. This nomenclature is consistent with that used in earlier MRI studies of body composition $(5,12)$. The muscle delineation is typically carried out by semi-manual boundary tracing techniques and is considered feasible albeit time consuming. Similarly, the extraction of subcutaneous fat can be achieved by tracing its internal surface. On the contrary, inter-muscular fat quantification is particularly challenging due to limitations in spatial resolution that blur the boundaries between the muscle and inter-muscular fat compartments. Therefore the automated quantification of muscle and adipose tissues is an active area of investigation. Earlier reports proposed semi-automated approaches that made use of simple histogram analysis and thresholding operations that typically had to be adjusted to compensate for inter-subject variability (7). Other notable works used fuzzy clustering followed by postprocessing (8), segmentation of muscle and fat from whole-body MRI using manual seeding (9), semi-automated intensity scaling (10), a registration-segmentation collaborative quantification scheme for the abdomen (11), and more advanced model-based segmentation and Expectation-Maximization histogram analysis applied to single-slice non-suppressed $T_{1^{-}}$ weighted images (12). Although these methods have produced acceptable results, there is still a need for an automated, fat and muscle quantification algorithm that can be applied to $3 \mathrm{D}$ volumetric datasets and that makes use of the improved muscle-fat separation provided by fat- and water-suppressed images.

We introduce here an automated method for segmentation and quantification of muscle and fat in cross-sectional MR images of the middle thigh. The goal is to provide an automated, accurate and reproducible approach to leg composition assessment. We propose that a joint multivariate analysis of water- and fat-suppressed $T_{1}$-weighted 3D MR images along with their non-suppressed counterparts will provide improved separation between muscle and fat voxels. Typical images are shown in Fig. 1. Our procedure is as follows. First, we extract the subcutaneous fat by applying a parametric deformable model to the 2D slices of the nonsuppressed volumetric dataset and produce a 3D intensity image that includes the muscle, intermuscular fat and bone. Next, we apply unsupervised clustering on the feature space defined by the water- and fat-suppressed image intensities of the 3D datasets and basic connected component shape feature ranking to separate the muscle from the bone and intermuscular fat, and remove the bone. To the best of our knowledge, the cluster analysis of samples in the joint feature domain of fat- and water-suppressed image intensities has not been previously reported in the literature.

In the validation stage, we compared the quantification results from our automated approach to a clinician-driven semi-manual commercial method that was applied to 2D CT images of the thigh obtained on the same participants. Even though the semi-manual CT-based analysis produces quite reasonable quantification of muscle and fat and was chosen as our reference in the validation experiments, it cannot be considered as a gold standard in the strict sense, since this would require an entirely manual quantification of the tested MR and histological imaging data. In this context, the goal of our experiments is to demonstrate agreement between laborious semi-manual quantification of 2D CT data and an automated method introduced herein and applied to 3D MR images. Our experiments suggest that the automated MRI-based fat and muscle quantification method meets the validation objectives. We also discuss certain inherent advantages and limitations in section 


\section{MATERIALS AND METHODS}

\section{MR Imaging Methods (Water-, fat-and non-suppressed images)}

The MR images were acquired on an Achieva 3T scanner (Philips Healthcare, Best, The Netherlands) using a Q-body coil. A rapid survey scan was obtained to identify axial slice locations, using the proximal and distal ends of the femur as landmarks. Next, three scans were acquired sequentially using a 3D multi-slice FFE pulse sequence. Repetition time (TR), echo time (TE), flip angle (FA), field of view (FOV), and number of excitations $(\mathrm{NEX})$ were: $\mathrm{TR} / \mathrm{TE}=25 / 3.8 \mathrm{msec}, \mathrm{FA}=30^{\circ}, \mathrm{FOV}=440 \times 300 \times 400 \mathrm{~mm}^{3}, \mathrm{NEX}=1$. The first scan was acquired without spectral suppression while the second and third scans were acquired with water suppression or fat suppression, respectively. This yields fat-and-water, fat-only and water-only images in the same spatial domain. The entire thigh was imaged with 805 -mm-thick slices with in-plane resolution of $0.98 \times 0.98 \mathrm{~mm}^{2}$. As dictated by the 3D acquisition protocol, there was no gap between the slices. The field of view encompassed both left and right thighs.

\section{Fat and Muscle Quantification}

Our algorithm can be divided into two main stages. First, we use a parametric deformable model to extract the subcutaneous adipose tissue from a non-suppressed $T_{1}$ weighted 3D image. We then identify the muscle and inter-muscular fat using unsupervised clustering. This process produces three masks corresponding to subcutaneous fat, inter-muscular fat and muscle. The main stages of our algorithm are depicted in Fig. 2 along with intermediate and final results after the application of each stage.

Parametric Deformable Models for Extraction of Subcutaneous Fat-The first step in our methodology is the delineation of the internal subcutaneous fat surface from the rest of the image. Limitations of spatial resolution resulting in partial voluming, and inhomogeneities of the radio frequency $\left(B_{1}\right)$ field unique to the MRI technique, complicate this process. As a result, simple morphological operations, or algorithms based on predicates of region uniformity such as region growing, produce inaccurate delineations. On the other hand, segmentation techniques that use parametric or geometric surface models have produced encouraging results in the context of medical imaging for delineation of body structures $(13,14,15,16)$. Desirable features of these techniques include their ability to process images possessing low contrast, high noise level and inhomogeneity of the $B_{1}$ field. Here we use a simple version of the parametric deformable model of (17) over multiple spatial scales. We generate a Gaussian pyramid that provides a multi-scale representation of the image content and we delineate the subcutaneous fat going from coarser to finer scales. The deformable model converges to a specific delineation at each step that is used to initialize the parametric contour in the next scale, therefore achieving faster and more accurate convergence than single scale processing.

Deformable models are dynamic models whose 2D and 3D shape evolves under the influence of internal and external forces. This evolution may be formulated as the minimization of an energy function that is divided into i) internal energy $E_{\text {int }}$ that maintains the regularity and continuity of the contour/surface ii) an image-based term $E_{\text {im }}$ that attracts the model to specific features of the image and iii) external energy $E_{\text {ext }}$ that incorporates $a$ priori knowledge and user interaction.

In parametric deformable models the active contour is represented by a set of points with initial position $\mathbf{u}(\mathbf{s})$. The energy function $\varepsilon$ to be minimized is given by 


$$
\mathscr{E}=\int_{0}^{1} E_{\text {int }}(\mathbf{u}(\mathbf{s}))+\kappa E_{\text {im }}(\mathbf{u}(\mathbf{s}))+\lambda E_{\text {ext }}(\mathbf{u}(\mathbf{s})) \mathbf{d} \mathbf{s}
$$

where

$$
E_{\text {int }}(\mathbf{u}(\mathbf{s}))=\mathbf{1} / \mathbf{2}\left(\alpha\left|\mathbf{u}^{\prime}(\mathbf{s})\right|^{2}+\beta\left|\mathbf{u}^{\prime \prime}(\mathbf{s})\right|^{2}\right)
$$

The internal energy terms defined in [2] are related to the contour's geometry and penalize stretching and bending while the image-based term pulls the snake towards edge points and the external term may be a probability map and/or a constant (inflating or deflating) balloon force. The energy-minimizing contour can be found by a solution of the Euler-Lagrange equation. This is accomplished by treating $\mathbf{u}$ as a function of time $t$ and position $\mathbf{s}$, i.e., $\mathbf{u} \equiv \mathbf{u}(\mathbf{s}, \boldsymbol{t})$ and solving [3] numerically.

$$
\frac{\partial \mathbf{u}(\mathbf{s}, t)}{\partial t}=\alpha \mathbf{u}^{\prime \prime}(\mathbf{s}, t)-\beta \mathbf{u}^{\prime \prime \prime}(\mathbf{s}, t)-\kappa \nabla E_{i m}-\lambda \nabla E_{e x t}
$$

The weighting parameters of the elasticity $\alpha$, rigidity $\beta$, image force $\kappa$ and external force $\lambda$ of the active contour model need to be experimentally tuned to address requirements posed by the user (such as delineation accuracy) or the application domain (imaging modality, tissue contrast and signal noise level). We apply the deformable model to the 2D slices of the nonsuppressed 3D image since it is less affected by intensity inhomogeneities than the waterand fat-suppressed sequences. In order to apply the snake segmentation we first select one leg and compute a parametric representation of the thigh periphery using conventional preprocessing operations. The legs are first separated from surrounding air using 2-class Otsu thresholding, after which the centroids of the two connected component regions corresponding to the legs are computed. We use the horizontal coordinates of these centroids to identify the left leg, then apply morphological erosion with a small structure element to the left leg ROI and sample the contour of the resulting region at a fixed interval to produce the initial set of points used in Eq. [1]. We then use a traditional gradient magnitude-based image force $\left(E_{i m}\right)$ in conjunction with a deflating external force $\left(E_{\text {ext }}\right)$ in order to attract the snake to the internal surface of the subcutaneous adipose tissue. An example of the active contour evolution is displayed in Fig. 3. Extraction of the subcutaneous fat is seen in (Fig. 4) after final convergence of the active contour to the boundary between the subcutaneous fat and muscle.

Detection of Muscle and Inter-muscular Fat-After the extraction of the subcutaneous fat, we proceed with the segmentation of the intermuscular fat and muscle. In the first step of this process we remove the bone region. We identify the cortical bone using multiple Otsu thresholding on the non-suppressed image without the subcutaneous fat and the background voxels to generate three groups: \{cortical bone\}, \{muscle\}, and \{intermuscular fat, bone marrow $\}$. The cortical bone is readily identified as the group with smallest intensity in the non-suppressed image. On the other hand, the bone marrow identification is more challenging because it includes mostly fat and cannot be distinguished from the inter-muscular fat by intensity alone. A solution is to use spatial connectivity and shape information, so we apply connected component labeling to the highest intensity group and compute the area and roundness of each component. After excluding components smaller than a pre-defined area threshold, we label as bone marrow the region with the maximum roundness. We remove the cortical bone and bone marrow regions from the mask 
that was generated by the snake segmentation to include only voxels belonging to muscle and inter-muscular fat.

We then apply K-means clustering $(18,19)$ to the samples defined in the joint space of water-suppressed and fat-suppressed intensities in order to divide the volumetric image into two clusters corresponding to fat and muscle. K-means belongs to the family of centroid clustering algorithms that seek to partition the data samples into $K$ disjoint groups based on their pairwise distances in the feature space. The objective is to minimize the distances within the clusters and maximize them between the different clusters. Each cluster is represented by its centroid. The K-means algorithm iteratively minimizes a score that is defined by the total inter-sample distances within each cluster until convergence. It is an unsupervised learning method, i.e., given a user-defined number of clusters, it groups the unlabeled data without a requirement for a training stage. We apply this procedure to the feature space defined by the voxel intensities of the fat- and water-suppressed images, which are less affected by partial voluming and therefore more amenable to clustering than the non-suppressed 3D image. Since we have already extracted the subcutaneous fat and the bone tissues, the voxel intensity samples of fat- and water-suppressed images should ideally form two clusters in the joint feature space that correspond to \{intermuscular fat $\}$ and \{muscle\}. A scatterplot of the fat and water intensity samples before and after labeling is displayed in Fig. 5. After clustering, the voxel labels are mapped back to the spatial 3D image domain producing muscle-only and inter-muscular-fat-only images. Orthogonal views of the segmented inter-muscular fat and muscle are shown in Fig. 6.

\section{RESULTS}

After acquisition, the datasets in DICOM format were transferred to an image analysis workstation. Datasets with poor SNR, imaging artifacts, or non-ideal slice placement were excluded. The number of participants before and after screening was 40 and 28 respectively. The test datasets included 17 females, and 11 males and the age ranges were $66.3 \pm 11.5$ years and $67.2 \pm 10.0$ years respectively. The 3D images obtained from these participants were pre-processed before further analysis. We first applied the N3 method (20) to remove intensity nonuniformity and then we linearly co-register the WS and FS images in the nonsuppressed reference space by use of (21) to address patient motion and facilitate their analysis in the joint feature space. We subsequently applied the proposed tissue composition analysis to produce 3D image masks corresponding to the subcutaneous fat, muscle, bone and inter-muscular fat with the same geometry as the input volumetric data, as seen in Fig. 7. Further, our algorithm computes total volumes of subcutaneous fat, muscle, and intermuscular fat as well as the histograms of signal intensity for these three regions over the non-suppressed, water- and fat-suppressed images. Limited parameter re-tuning was required to address differences in the thickness of the subcutaneous fat compartment between the male and female participants.

We validated this method against muscle and fat tissue composition measures from single slice CT datasets of the same participants. The CT datasets were previously analyzed by a clinical specialist who used a semi-manual approach provided by Geanie software (Bonalyse Oy, Jyvaskyla, Finland). Specifically, $10 \mathrm{~mm}$ axial slices were obtained at the mid-thigh using a Siemens Somatom Sensation 10 CT scanner (Siemens, Malvern, PA). Images were reviewed to verify correct positioning, symmetry, absence of artifacts and quality using Image $\mathbf{J}$ (NIH, Bethesda, MD). The Geanie software was used to analyze each CT thigh image for muscle characteristics, i.e., the cross sectional area of the muscle $\left(\mathrm{mm}^{2}\right)$, density of the muscle $\left(\mathrm{mg} / \mathrm{cm}^{3}\right)$, and cross sectional area of fat $\left(\mathrm{mm}^{2}\right)$. The left thigh image was analyzed for each participant. On each image the clinician manually outlined the region of interest to be analyzed by the program. The exterior surface of the thigh was traced and the 
femur was manually delineated in order to exclude it from the analysis. The computed features were the muscle and total fat areas.

In order to establish a common basis for our inter-modal comparisons we applied our automated method to the mid-thigh slices of our MRI datasets that cover approximately the same region as the CT slice used in the semi-manual analysis. The computed volumes from MRI were then averaged and divided by the MRI voxel height (i.e., the slice thickness) to obtain the corresponding areas. These quantities were then compared with the areas computed from CT. The image quantification methodology was implemented in MATLAB. The typical execution time for 3 slices was approximately $55 \mathrm{sec}$ on a linux-based platform with one quadruple core Intel CPU at $2.33 \mathrm{GHz}$ and 4 GBytes of RAM without applying specific optimization for speed in this software implementation.

We computed the degree of consistency between the semi-manual CT-based and the automated MRI-based approaches using group statistics. First, we computed the Pearson correlation coefficient $\rho$ between the areas of muscle and fat from the two techniques to measure the degree of linear correlation between the two measurements. Furthermore, we computed the subject-wise relative differences between the area measures and estimated their mean. The next step was to compute $\mathrm{p}$-values from the $\chi$-squared test to determine whether there was systematic correlation between the two methods. Also, we used the coefficient of variation $(\mathrm{CV})$ to estimate the dispersion of the absolute area differences between the automated and the semi-manual methods.

In addition, we compared results obtained by the joint K-means clustering analysis of the water- and fat-suppressed images described above with results produced by a more standard analysis of the non-suppressed images only. To do this we used as input to our clustering algorithm the non-suppressed image intensities only, left the other stages of the methodology unchanged and repeated the above experiments. These results are reported in Fig. 8 and Table 1. Fig. 8 displays scatterplots of the muscle and fat areas computed by joint analysis of the water-suppressed and fat-suppressed images, or the non-suppressed data only, versus the reference method. The slope and intercept coefficients obtained from linear fitting are $[0.98,481]$ and $[0.96,591]$ for the fat and muscle regions respectively using the joint analysis. The corresponding coefficients when using the non-suppressed images only are $[0.9,1890]$ and $[0.99,117]$. The slope coefficients were close to 1 , while the intercepts indicated slight over-estimation of the muscle. We note here that the non-suppressed-only analysis considerably overestimates the fat as indicated by the larger intercept value and the deviation of slope from 1. Furthermore, from Table 1 we observe that the average relative differences between the MRI-based and CT-based techniques for muscle and fat were 4.39\% and $5.74 \%$ when using the joint analysis and $7.75 \%$ and $15 \%$ for the non-suppressed-only analysis, so the joint analysis of WS and FS images reduces the relative error by $43 \%$ and $62 \%$ for the muscle and fat areas respectively. The Pearson correlation coefficients were greater than 0.97 for both measured areas when using joint WS and FS analysis, and the $C V \mathrm{~s}$ of the absolute differences were very low, so both $\rho$ and $C V$ indicated very good correlation. The $\chi$-squared tests yielded $P<10^{-30}$ confirming a deterministic relation between the muscle and fat areas computed by the proposed approach and the corresponding measures obtained by semi-manual processing of the CT images.

\section{DISCUSSION}

We have introduced a new method for fat and muscle quantification based on water- and fatsuppressed MR images. The first goal was to validate quantification accuracy by comparison with a semi-automated method based on CT data. The second goal was to underline the advantages of joint water-suppressed and fat-suppressed analysis in comparison with the 
more standard analysis using only non-suppressed MR images. While a fully rigorous validation would require the use of a gold standard produced by manual quantification of volumetric MRI datasets and computation of overlap measures, our correlation analysis indicated that the proposed method produces results consistent with the reference semimanual procedure applied to the CT data. Furthermore, we consider the results of our methodology to be particularly encouraging for the following reasons: i) The proposed method is automated and therefore reproducible and time efficient, while the semi-manual approach requires a trained user to draw one contour that circumscribes the skin surface and another contour that circumscribes the bone; ii) our method is considerably insensitive to anatomical and image quality differences between the participants; and iii) our method can be readily applied to volumetric datasets to produce robust estimates of local body composition. On the contrary, the semi-manual CT-based method operates on a single slice, and is therefore dependent on the slice location and anatomical position as reported in (5).

iv) The ability to generate fat- and water- suppressed images is unique to the MRI approach, which produces better soft tissue contrast and is intrinsically safer than CT since it does not involve ionizing radiation; and $\mathrm{v}$ ) the proposed technique divides the fat tissue into subcutaneous and inter-muscular compartments that cannot be separated by the semi-manual CT-based methodology of Geanie software version 2.1.

A key element of this work in comparison to the state of the art is the joint analysis of waterand fat-suppressed images. Our experiments suggest that the use of these tissue-specific images further improves the accuracy of tissue decomposition compared to the use of nonsuppressed images only. This improvement is especially evident in the identification of fat. One source of inaccuracy in segmentation based on non-suppressed MR images is the presence of the water-fat chemical shift artifact. This mixes water and fat intensities in the read direction of the MR image. In order to minimize this effect, high-bandwidth sampling is typically used; this is especially important at higher magnetic field strengths. However, such sampling decreases the image signal-to-noise ratio, introducing another source of inaccuracy. In contrast, use of water- and fat-suppressed images for segmentation avoids chemical shift artifacts, since the resulting images represent fat-only and water-only intensity respectively. This permits lower bandwidth sampling, and accordingly, allows for analysis of higher SNR images (22). Further, the enhanced separation between the intensities of muscle and fat voxels in the tissue-specific domain was found to facilitate the cluster analysis on the global scale.

While effective and efficient, certain difficulties remain with the described algorithm. The detection of inter-muscular fat is challenging. One reason for this is the partial volume effect which manifests itself as diffuse boundaries between intermuscular fat and the muscle compartments. In addition, the signal intensities in fat- and water-suppressed images are correlated, resulting in non-spherical clusters in the joint feature space which may not be appropriately approximated using centroid clustering models such as K-means clustering. Consequently, alternative clustering methods that use parametric (e.g., mixtures of Gaussians) or non-parametric (e.g., Parzen-based) probability density models may exhibit greater effectiveness for these datasets.

Finally, with respect to body composition analysis, we note an intrinsic limitation of MR as compared to CT investigations of tissue. MR image intensities are non-linear functions of tissue composition and therefore cannot readily be used to estimate muscle tissue density. In contrast, CT image intensity provides a direct assessment of the water-to-fat ratio. This is an important measure, serving as a proxy for muscle quality because it reflects microscopic fat infiltration (23). However, Dixon imaging techniques in MRI $(24,25)$ may be used to compute the ratio of water to fat within individual imaging voxels, which may serve as an implicit index of muscle quality. 
In conclusion, we present an automated method for quantification of fat and muscle in the thigh through the use of non-suppressed, water- and fat-suppressed images. The method includes an active contour model for segmentation of the subcutaneous fat followed by cluster analysis for detection of inter-muscular fat and muscle. We validated our quantification results against area measures that were computed from 2D CT imaging data on the same participants using a semi-manual approach operated by a clinical specialist. Our findings suggest that the muscle and fat quantification results produced by the proposed MRI-based method are in good agreement with those produced by the semi-manual CT method, especially considering the differences in physical properties, image contrast, geometry and field of view between these two imaging techniques.

\title{
ABBREVIATIONS
}

\author{
NS Non-Suppressed \\ FS Fat-Suppressed \\ WS Water-Suppressed \\ CV Coefficient of Variation
}

\section{References}

1. Goodpaster BH, Thaete FL, Simoneau JA, Kelley DE. Subcutaneous abdominal fat and thigh muscle composition predict insulin sensitivity independently of visceral fat. Diabetes. Oct; 1997 46(10):1579-1585. [PubMed: 9313753]

2. Fishbein MH, Mogren C, Gleason T, Stevens WR. Relationship of hepatic steatosis to adipose tissue distribution in pediatric nonalcoholic fatty liver disease. J Pediatr Gastroenterol Nutr. Jan; 2006 42(1):83-88. [PubMed: 16385259]

3. Flegal KM, Carroll MD, Kuczmarski RJ, Johnson CL. Overweight and obesity in the United States: prevalence and trends, 1960-1994. Int J Obes Relat Metab Disord. Jan; 1998 22(1):39-47. [PubMed: 9481598]

4. Ferrucci L, Studenski S. Diabetes, muscles, and the myth of Ulysses' bow. Diabetes Care. Nov; 2009 32( 11):2136-2137. [PubMed: 19875609]

5. Ruan XY, Gallagher D, Harris T, Albu J, Heymsfield S, Kuznia P, Heshka S. Estimating whole body intermuscular adipose tissue from single cross-sectional magnetic resonance images. J Appl Physiol. Feb; 2007 102(2):748-754. [PubMed: 17053107]

6. Schick F, Machann J, Brechtel K, Strempfer A, Klumpp B, Stein DT, Jacob S. MRI of muscular fat. Magn Reson Med. Apr; 2002 47(4):720-727. [PubMed: 11948733]

7. Colin, A.; Erbland, E.; Datin, C.; Boire, JY.; Veyre, A.; Zanca, M. Automatic muscle/fat quantification on MR images. Proc. IEEE 17th Annual Conference Engineering in Medicine and Biology Society; 1995; 1995. p. 479-480.

8. Barra V, Boire JY. Segmentation of fat and muscle from MR images of the thigh by a possibilistic clustering algorithm. Computer Methods and Programs in Biomedicine. Jun; 2002 68(3):185-193. [PubMed: 12074845]

9. Jin, Y.; Imielinska, C.; Laine, A.; Udupa, JK.; Shen, W.; Heymsfield, SB. Segmentation and evaluation of adipose tissue from whole body MRI scans. In: Ellis, RE.; Peters, TM., editors. MICCAI. Vol. 2878. Springer; 2003. p. 635-642.Lecture Notes in Computer Science

10. Mattei J, Fur Y, Cuge N, Guis S, Cozzone P, Bendahan D. Segmentation of fascias, fat and muscle from magnetic resonance images in humans: the dispimag software. MAGMA Magnetic Resonance Materials in Physics, Biology and Medicine. Nov; 2006 19(5):275-279.

11. Leinhard, OD.; Johansson, A.; Rydell, J.; Smedby, O.; Nystrom, F.; Lundberg, P.; Borga, M. Quantitative abdominal fat estimation using MRI. Proc. 19th International Conference on Pattern Recognition ICPR; 2008; 2008. p. 1-4. 
12. Positano V, Christiansen T, Santarelli MF, Ringgaard S, Landini L, Gastaldelli A. Accurate segmentation of subcutaneous and intermuscular adipose tissue from MR images of the thigh. $\mathrm{J}$ Magn Reson Imaging. Mar; 2009 29(3):677-684. [PubMed: 19243051]

13. Kass M, Witkin AP, Terzopoulos D. Snakes: Active contour models. International Journal of Computer Vision. Jan; 1988 1(4):321-331.

14. Chan TF, Vese LA. Active contours without edges. IEEE Transactions on Image Processing. Feb; 2001 10( 2):266-277. [PubMed: 18249617]

15. Malladi R, Sethian JA, Vemuri BC. Shape modeling with front propagation: A level set approach. IEEE Transactions on Pattern Analysis and Machine Intelligence. Feb; 1995 17(2):158-175.

16. Makrogiannis, S.; Bhotika, R.; Miller, JV.; Skinner, J.; Vass, M. Nonparametric intensity priors for level set segmentation of low contrast structures. In: Yang, GZ.; Hawkes, DJ.; Rueckert, D.; Noble, JA., editors. MICCAI. Vol. 5761. Springer; 2009. p. 239-246.Lecture Notes in Computer Science0002 CJT

17. Xu C, Prince JL. Snakes, shapes, and gradient vector flow. IEEE Transactions on Image Processing. Mar; 1998 7( 3):359-369. [PubMed: 18276256]

18. Duda, RO.; Hart, PE.; Stork, DG. Pattern Classification. Wiley-Interscience Publication; 2000.

19. Jain AK, Murty MN, Flynn PJ. Data clustering: a review. ACM Comput Surv. Sep; 1999 31(3): 264-323.

20. Sled JG, Zijdenbos AP, Evans AC. A nonparametric method for automatic correction of intensity nonuniformity in mri data. IEEE Trans Med Imaging. Feb; 1998 17(1):87-97. [PubMed: 9617910]

21. Jenkinson M, Smith S. A global optimisation method for robust affine registration of brain images. Med Image Anal. Jun; 2001 5(2):143-156. [PubMed: 11516708]

22. Mitchell, MG.; Cohen, MS. MRI Principles. Saunders; Philadelphia, Pa: 2004.

23. Goodpaster BH, Thaete FL, Kelley DE. Thigh adipose tissue distribution is associated with insulin resistance in obesity and in type 2 diabetes mellitus. Am J Clin Nutr. Apr; 2000 71(4):885-892. [PubMed: 10731493]

24. Dixon WT. Simple proton spectroscopic imaging. Radiology. Oct; 1984 153(1):189-194. [PubMed: 6089263]

25. Berglund, Johan; Johansson, Lars; Ahlström, Håkan; Kullberg, Joel. Three-point dixon method enables whole-body water and fat imaging of obese subjects. Magn Reson Med. Jun; 2010 63(6): 1659-1668. [PubMed: 20512869] 


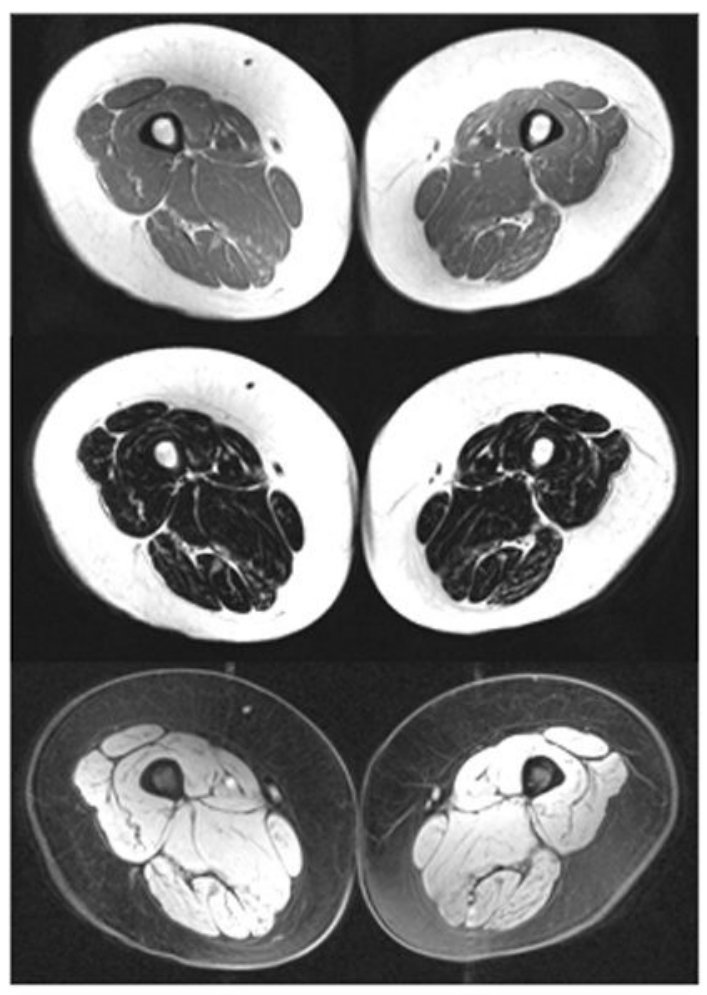

Fig. 1.

Examples of cross-sections (top to bottom) from non-suppressed, water-suppressed (fatonly) and fat-suppressed (water-only) MR volumetric datasets acquired from the same participant. Non-suppressed image: note the femur, surrounded by muscle compartments delineated by fascial layers and infiltrated with muscular fat. The subcutaneous fat is seen as the bright band surrounding the muscle. Water-suppressed image: note the brightness of the subcutaneous and intra-muscular fat intensities and loss of signal from the muscle. Fat suppressed image: note the loss of the subcutaneous and intra-muscular fat signal with preserved detail from the muscle. These are used as input for the muscle/fat quantification algorithm. The intensity inhomogeneity (shading) artifacts that are characteristic of high field MRI can be successfully addressed by the proposed approach. 


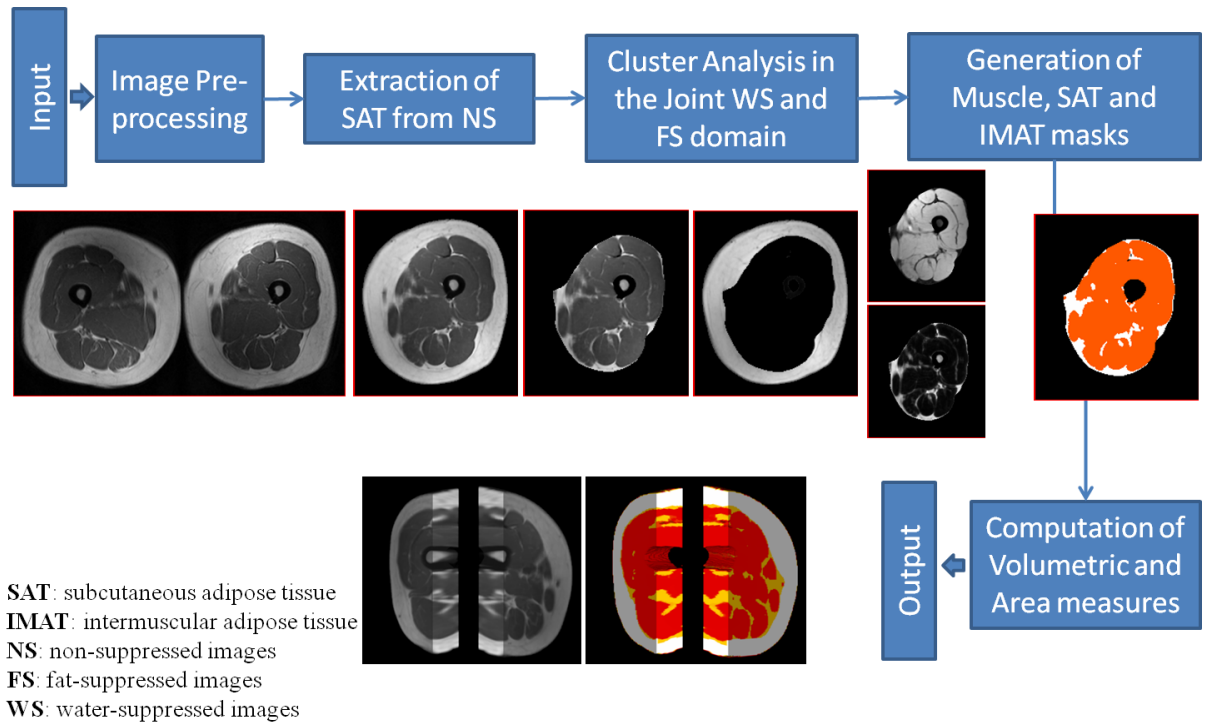

Fig. 2.

Flow chart of the image quantification algorithm. The 3D images are first cropped, linearly co-registered and the left thigh is automatically selected. Next, the subcutaneous fat is extracted by snake segmentation applied to the non-suppressed volumetric image and we map the extraction mask to the water-suppressed and fat-suppressed images. After removing the bone, we apply clustering of the remaining voxels in the joint fat-suppressed and watersuppressed feature space. Finally, we apply this 3D segmentation to the input nonsuppressed data set to generate subcutaneous fat, muscle, and intermuscular fat volumetric images. 


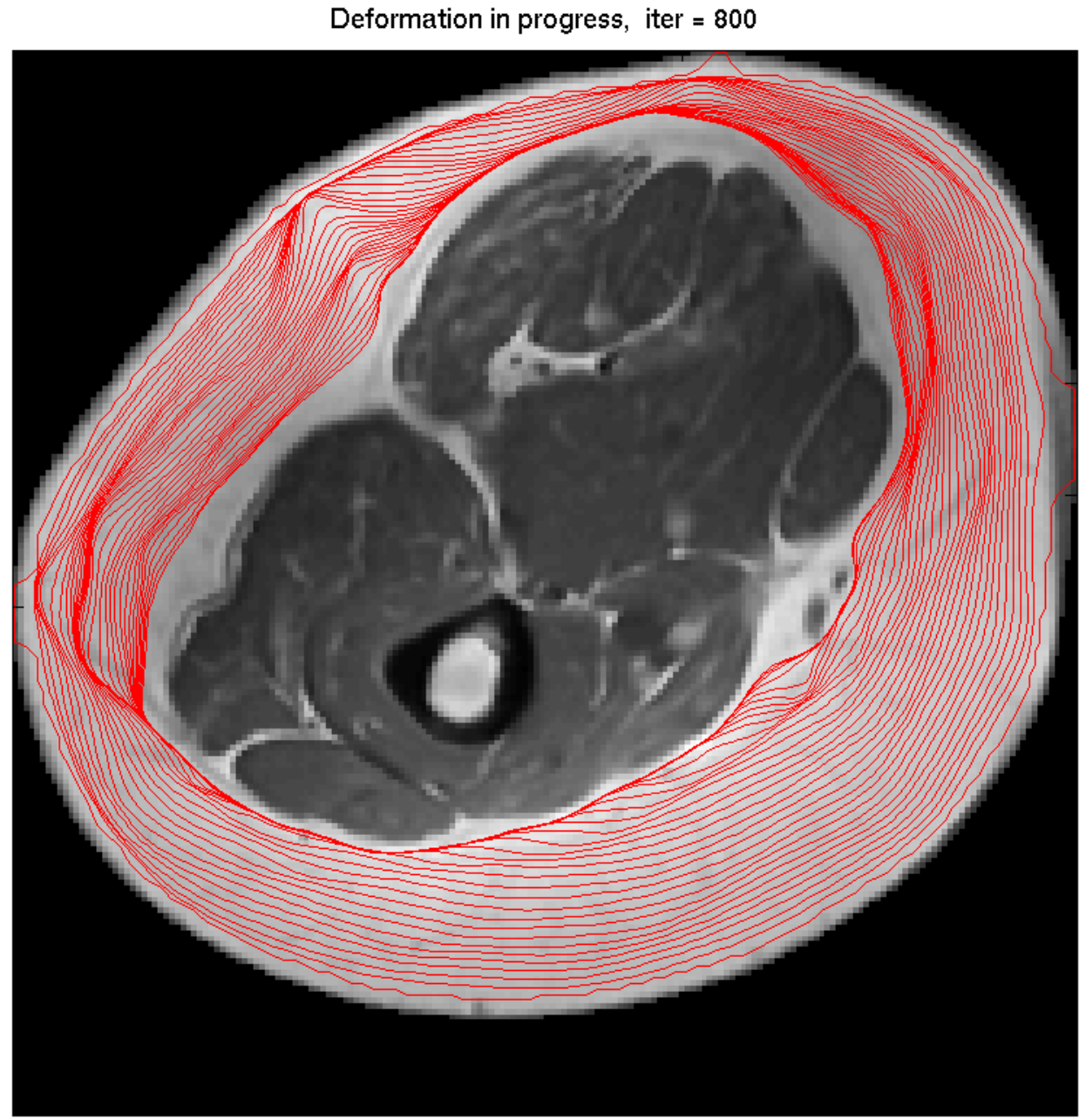

Fig. 3.

Example of convergence of the active contours for extraction of subcutaneous fat. The active contour evolves from the interior skin surface towards the boundary between subcutaneous fat and muscle. The final number of iterations is 800 . 

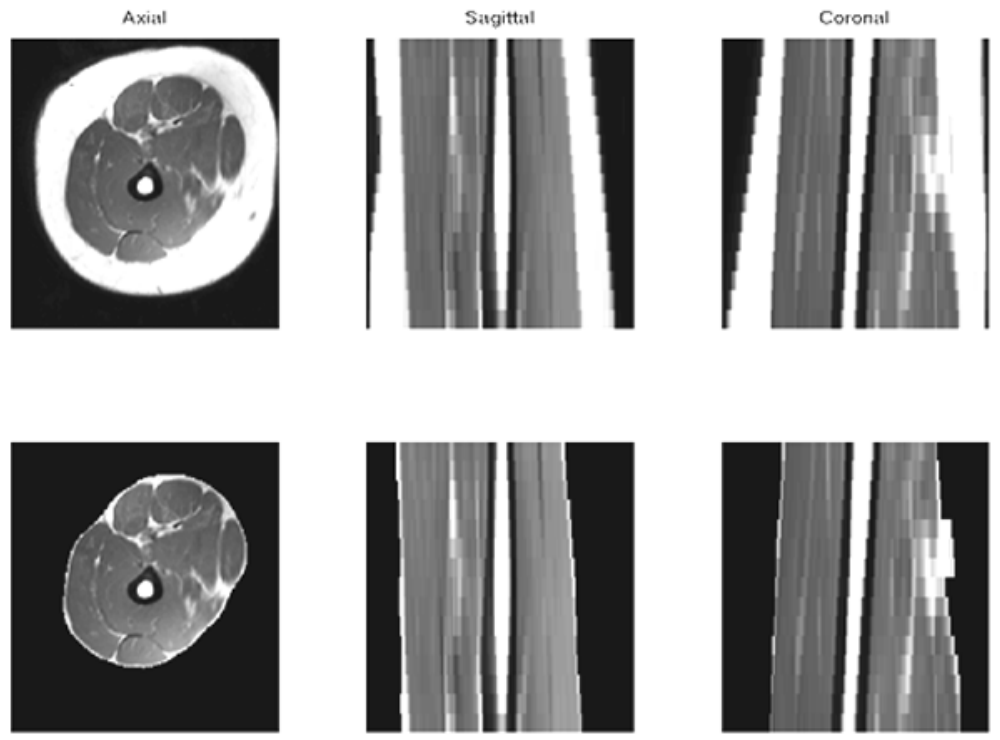

Fig. 4.

Orthogonal views of the original non-suppressed volumetric dataset (top row) and the algorithm output after selecting one leg and extracting the subcutaneous fat using active contour segmentation (bottom row). 


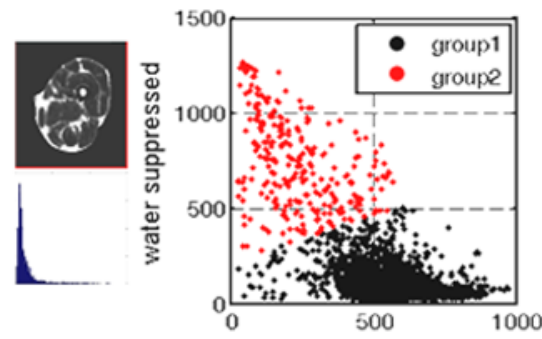

fat suppressed
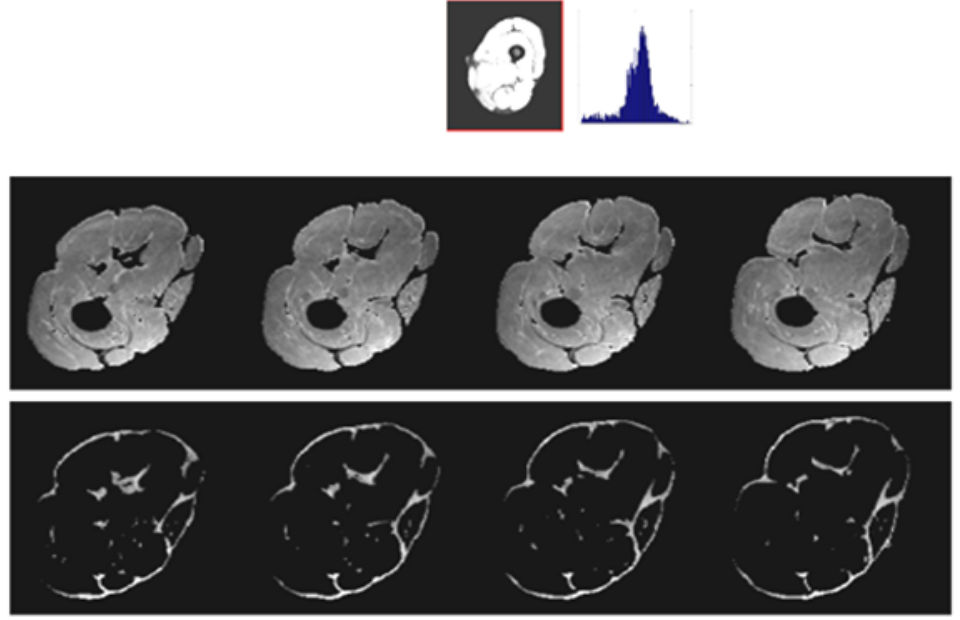

Fig. 5.

Scatter plots and marginal histograms of water- and fat-suppressed voxel intensities from the muscle and intermuscular fat regions after centroid clustering. The labels "group 1" and "group 2" correspond to clusters of \{muscle\} and \{intermuscular fat $\}$ samples, respectively. In the bottom part are depicted the corresponding muscle and intermuscular fat regions after re-mapping them onto the 3D image plane. 

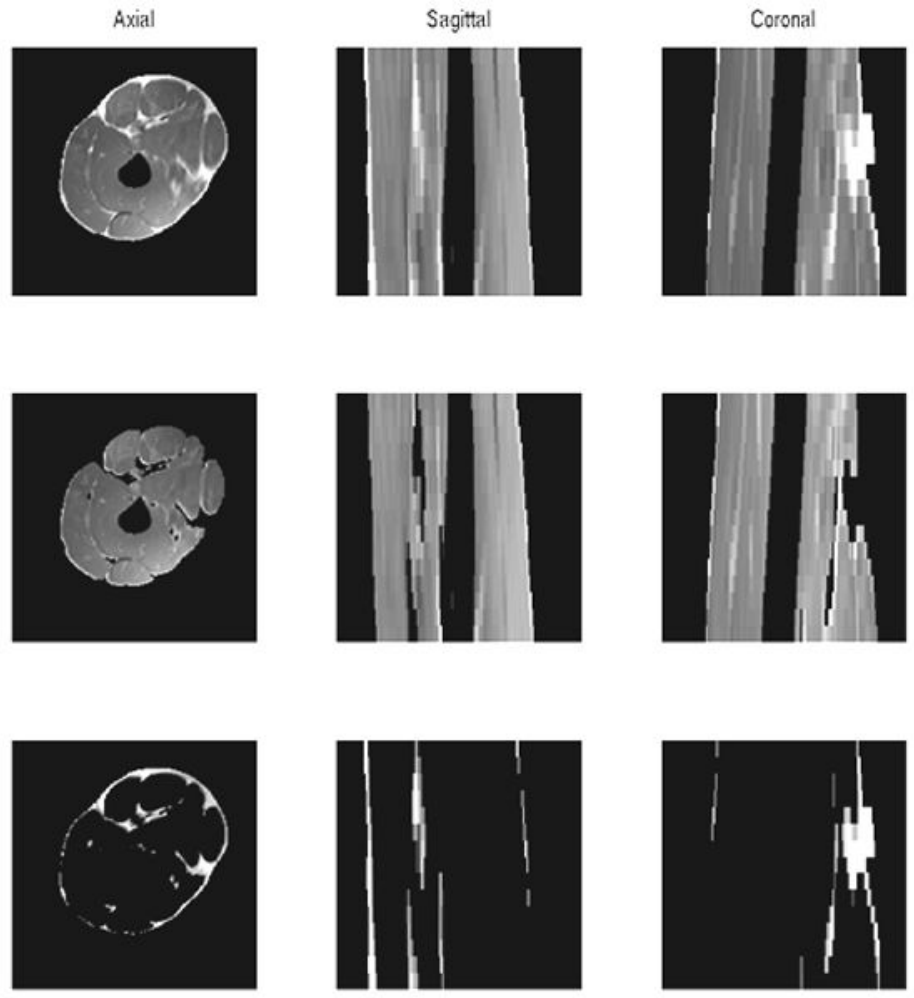

Fig. 6.

Orthogonal views after separation of muscle from inter-muscular fat using cluster analysis and connected component analysis to remove the bone. The top row shows the nonsuppressed $T_{1}$-weighted 3D image after the removal of subcutaneous adipose tissue and the femur, the middle row displays the segmented 3D image of muscle after extraction of intermuscular fat and the bottom row shows the segmented 3D image of the inter-muscular fat. 


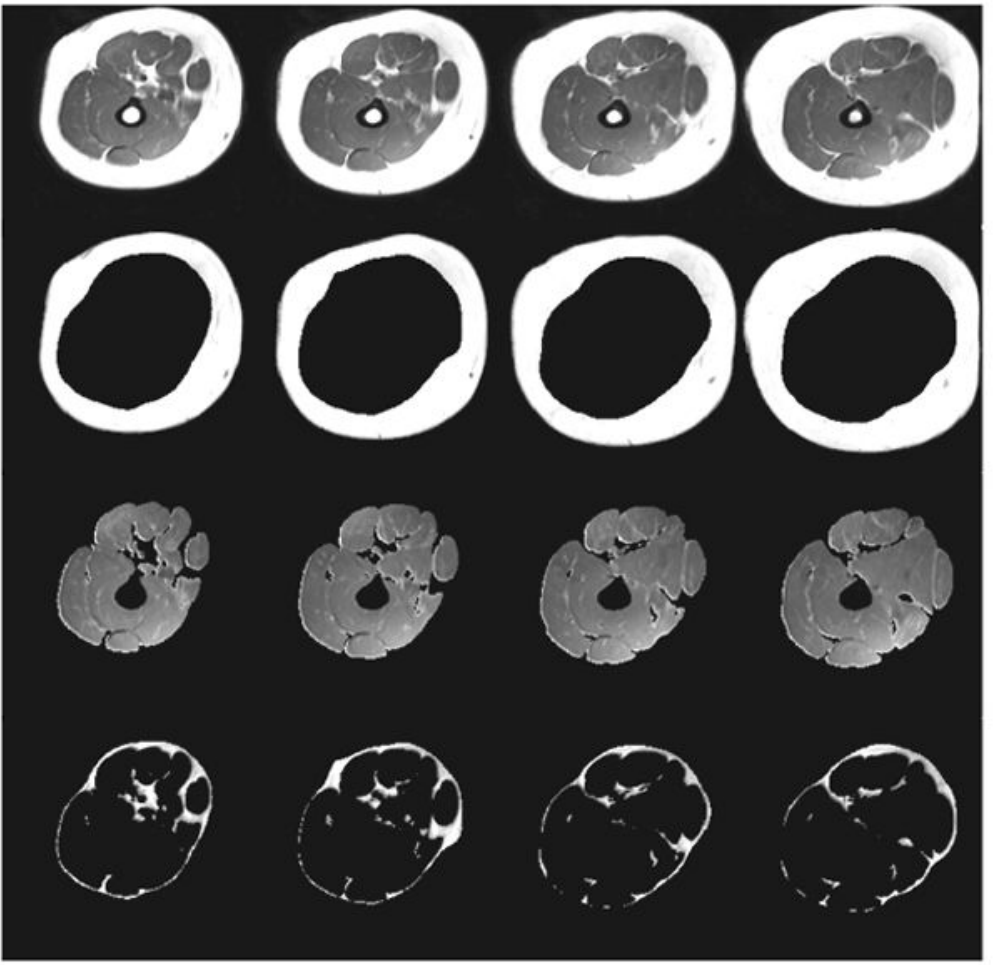

Fig. 7.

Multiple axial slices from the original non-suppressed volumetric dataset (top row) and its decomposition into subcutaneous fat, muscle and inter-muscular fat components (in the second, third and fourth row respectively). 

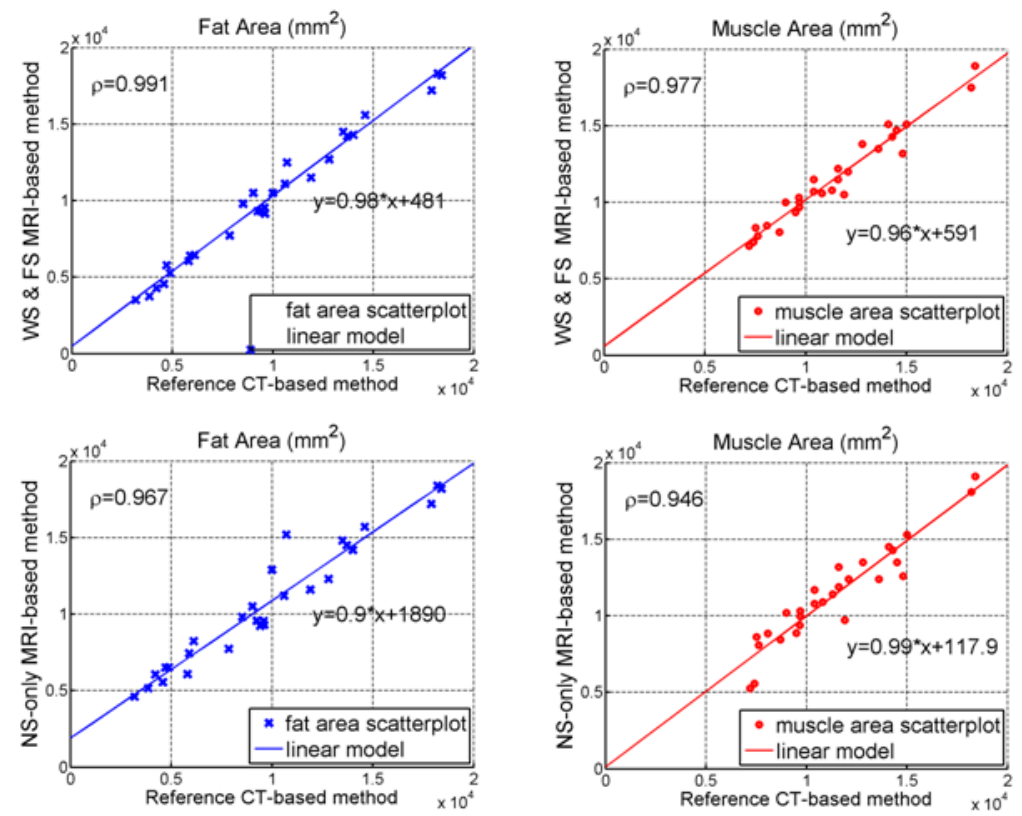

Fig. 8.

Scatterplots of the computed fat and muscle areas using the automated MRI-based method versus the semi-manual CT-based approach using the water- and fat-images (top row) or the non-suppressed images only (bottom row). 
Table 1

Statistical measures of agreement between the semi-manual CT- and the automated MRI-based fat and muscle quantification methods using the water- and fat-suppressed images (WS \& FS) and the non-suppressed (NS) images only $(\mathrm{N}=28)$.

\begin{tabular}{|c|c|c|c|c|}
\hline \multirow{2}{*}{ Measure } & \multicolumn{2}{|c|}{ Muscle Area } & \multicolumn{2}{|c|}{ Fat Area } \\
\hline & $W S \& F S$ & $N S$ & $W S \& F S$ & $N S$ \\
\hline Ave. Rel. Diff. between CT and MRI (\%) & 4.39 & 7.75 & 5.74 & 15.5 \\
\hline Pearson Corr. Coef. $(\rho)$ & 0.977 & 0.946 & 0.991 & 0.967 \\
\hline Coefficient of Variation (CV) & 0.89 & 0.83 & 0.96 & 0.92 \\
\hline
\end{tabular}

